\title{
Restoration of central macular pigment dip with dietary RR zeaxanthin supplementation in patients with AMD
}

\begin{abstract}
Objective: Low macular pigment optical density (MPOD) is a major risk factor for agerelated macular degeneration (AMD). This paper investigates the impact of $8 \mathrm{mg}$ dietary RR zeaxanthin (Z) supplementation on MPOD distribution and the associated effect on macular scotomas in patients with a central foveal dip in their macular pigment spatial profile.

Methods: This is a retrospective review of The Zeaxanthin and Visual Function RCT (FDA IND 78,973) with respect to the relation between low levels of MPOD and increased risk of age-related macular degeneration (AMD). We retrospectively examine the effect of daily supplementation of $8 \mathrm{mg}$ of dietary $\mathrm{Z}$ on $\mathrm{n}=4$ patients having a baseline central foveal dip in their MPOD distribution. The testing modalities used were a $7^{0} 3 \mathrm{D}$ specular reflectance and the associated kinetic $20^{\circ}$ macular visual fields.
\end{abstract}

Results: Over a 12-month period, all four patients showed a progressive increase in central peak restoration with related kinetic and/or Amsler grid visual field data.

Conclusion: The initial results demonstrate marked improvement in macular pigment architecture and overall visual function after 12 months of $Z$ supplementation. These results warrant a prospective clinical trial with a larger number of subjects to investigate the role of $Z$ in central dip amelioration.

Keywords: zeaxanthin, meso-zeaxanthin, amd, central foveal dip, scotomas, macular pigment optical density
Volume 7 Issue 3 - 2017

\author{
Stuart P Richer, ${ }^{1,2,3}$ Brian Cebold, ${ }^{1,2}$ Mansi \\ Katkar, ${ }^{1,2}$ Byki Huntjens, ${ }^{3}$ Steven G Pratt, ${ }^{4}$ \\ William Stiles, ${ }^{5}$ Lawrence Ulanski,' Dennis \\ Gierhart ${ }^{5}$ \\ 'Captain James A Lovell Federal Health Care Center, USA \\ ${ }^{2}$ Chicago Medical School, Rosalind Franklin University of \\ Medicine and Science (RFUMS), USA \\ ${ }^{3}$ Scripps Health/Scripps Memorial Hospital/Scripps Mericos Eye \\ Institute, USA \\ ${ }^{4}$ Applied Vision Research Centre, University of London, UK \\ ${ }^{5}$ ZeaVision LLC, 680-F Crown Industrial Court Chesterfield, \\ USA
}

Correspondence: Stuart P Richer, Captain James A Lovell Federal Health Care Facility, 300I Green Bay Rd, North Chicago, Illinois, 60064 USA, Email Stuart.Richer I @va.gov

Received: July 14, 2017 | Published: July 28, 2017
Abbreviations: L, lutein; Z, rr zeaxanthin; Mz, mesozeaxanthin (rs zeaxanthin); AMD, age-related macular degeneration; MPOD, macular pigment optical density

\section{Introduction}

The central macula lutea (fovea) contains the highest density of cone photoreceptors, responsible for detailed and color vision in the central $2-3^{\circ}$ of the visual field. AMD is the leading cause of permanent vision impairment in aging Western populations, with the Centers for Disease Control and Prevention estimating that 1.8 million Americans over age 40 have AMD and another 7.3 million Americans have large drusen, which places them at a high risk of developing AMD. ${ }^{1}$ Research demonstrates that the relationship between MPOD and central retinal thickness has a strong positive correlation, ${ }^{2}$ and additional studies show that reduced MPOD is associated with a higher risk of developing AMD. ${ }^{3}$ Heterochromatic flicker photometry has been used to measure the spatial profile of MPOD, with two predominant profile types emerging: typical exponential/peaked profile or atypical "central dip" profile. ${ }^{4}$ The MARS study by Dietzel et al., ${ }^{5}$ found $19.8 \%$ of subjects possess this central dip profile, meaning they demonstrate a significantly lower MPOD at retinal eccentricities of $0^{\circ}, 0.25^{\circ}$, and $0.5^{\circ}$ but significantly higher MPOD at $1^{\circ}$ and $2^{\circ}$ when compared to subjects with no central dip. Concordance between pairs of eyes was labeled as highly significant. ${ }^{5}$

The underlying cause of this central dip profile is not well understood, though research showing a higher incidence in certain ethnic populations suggests a genetic link. ${ }^{6,7}$ The three primary risk factors for MPOD central dip have been identified as the same three primary risk factors for AMD: age, smoking, and elevated BMI. ${ }^{4,8}$ Macular pigment is composed of three carotenoids: lutein (L), RR zeaxanthin (Z), and RS meso-zeaxanthin (Mz). These three molecules are collectively known as xanthophyll carotenoids or macular carotenoids. $\mathrm{L}$ and $\mathrm{Z}$ have both been well-studied and are found in many vegetables regularly consumed by humans such as spinach, broccoli, kale, and bell peppers. ${ }^{9}$ The role of $\mathrm{Mz}$ is not as well understood: it has recently been found in the human food chain in the skin of a handful of species of fish, but only in trace quantities. Research has shown that Mz can be enzymatically derived from $\mathrm{L},{ }^{10}$ though the relative roles of endogenous $\mathrm{Mz}$ isomerization versus dietary consumption of $\mathrm{Mz}$ in retinal health remain unstudied (Figure $1-3)$.

Studies have shown that macular carotenoid supplementation provides myriad visual health benefits, including improved visual acuity, glare recovery, and contrast sensitivity function ${ }^{11}$, along with increasing both neural processing speed and efficiency of visual signals. ${ }^{12}$ Furthermore, macular carotenoid supplementation has been linked to improved cognitive function ${ }^{13}$ and reduced cortisol levels. ${ }^{14}$ The ratios of $\mathrm{L}, \mathrm{Z}$, and $\mathrm{Mz}$ vary throughout the macula, though total MPOD peaks in the central fovea/foveola. Dietary $\mathrm{Z}$ prevents oxidative stress by absorbing harmful blue light $(400-500 \mathrm{~nm}),{ }^{15}$ which reduces retinal inflammation and neovascularization within the photoreceptive/RPE layers. $\mathrm{Z}$ supplementation has been shown to increase MPOD and improve visual function, ${ }^{16,17}$ yet there has been no research on the impact of macular carotenoid supplementation on the 
volumetric profile of macular pigment, particularly the central foveal dip. This article aims to investigate the effect of $\mathrm{Z}$ supplementation over a 12-month period on patients with central foveal dip by measuring serial MPOD and corresponding $20^{\circ}$ central macular visual field scotoma resolution.

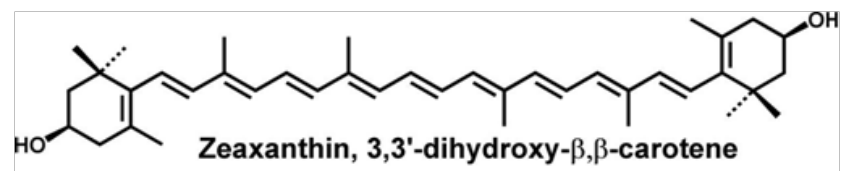<smiles>CC1=C(/C=C/C(C)=C/C=C/C(C)=C/C=C/C=C/C(C)=C/C=C/C=C(C)/C=C/C2=C(C)C[C@@H](O)CC2(C)C)C(C)(C)CC(O)C1</smiles><smiles>CC1=C[C@H](O)CC(C)(C)[C@H]1/C=C/C(C)=C/C=C/C(C)=C/C=C/C=C(C)/C=C/C=C(C)/C=C/C1=C(C)CC(O)CC1(C)C</smiles>

Figure I Molecular structures of the three macular carotenoids: Zeaxanthin, Meso-zeaxanthin, and Lutein.

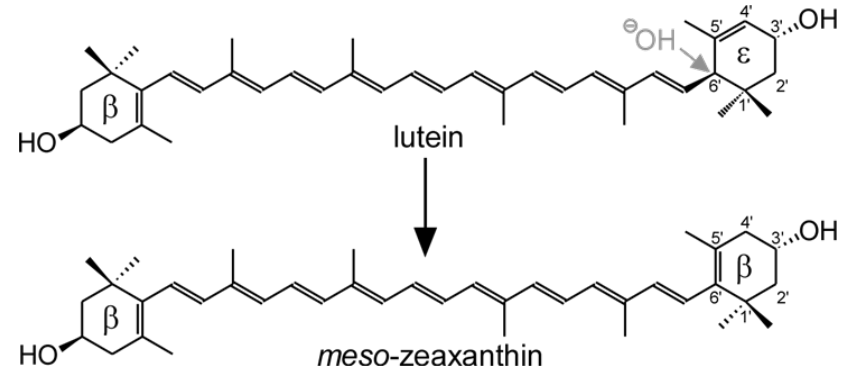

Figure 2 Enzymatic conversion of Lutein to Meso-Zeaxanthin.

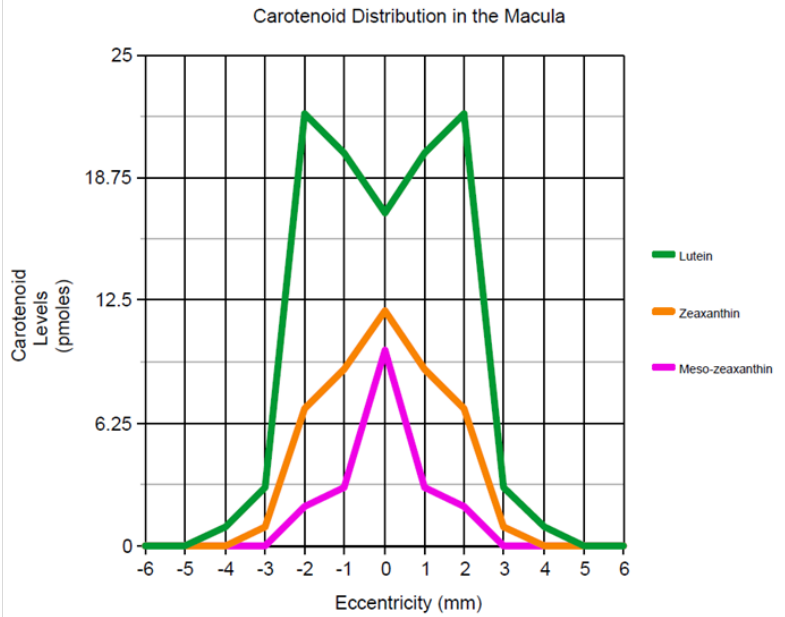

Figure 3 Distribution of macular carotenoids as a function of retinal eccentricity. ${ }^{40}$

\section{Methods}

The data represents individual subjects within the ZVF study (Zeaxanthin and Visual Function), a 1-year prospective, doublebind RCT at the DVA North Chicago Medical Center. ${ }^{17}$ Recruitment was staggered and began on November 26, 2007. The final subject completed his 12-month evaluation on May 19, 2009. The ZVF Study received approval from the DVA Office of Research and Development and Human Subjects Protection (ZVF-FDA IND \#78, 973) and was registered with www.clinicaltrials.gov. The study included 60 subjects with early and moderate AMD assigned randomly into 3 groups, each receiving supplementation consisting of: $8 \mathrm{mg}$ of $Z \quad(n=25)$, a combination of 8-mg Z plus $9 \mathrm{mg} \mathrm{L}(\mathrm{n}=25)$, or a "faux placebo" 9-mg L supplement $(n=10)$. Subjects were excluded from the study if they either manifested or displayed high-risk retinal characteristics of advanced AMD, for which medical or surgical options for treatment were available. The Automated Retinal Imaging System ${ }^{\circledR}$ (ARIS, Prescott, AZ, USA) used a specular reflectance technique to acquire the spatial distribution of macular pigment. A $7^{\circ}$, 3-dimensional image of 3D macular pigment distribution was obtained through ARIS using an auto fluorescence technique. ${ }^{17}$ The macular visual fields were evaluated using the SimulEyes ${ }^{\circledR}$ Kinetic Visual Field (Gold Beach, OR, USA). The testing software evaluated monocular central field vision with best refractive correction at each visit. This visual field instrument incorporates an area-integrating, touch screen monitor that displays a central fixation point and movable horizontal or vertical raster lines. This allows subjects to more precisely outline the boundaries of their scotoma in comparison to an Amsler grid. Additionally, spectral separation diminishes media influences such as confounding age-related cataract. Scotomas within the central 20 -degree visual field were assessed at 5 contrast levels $(20,40,60,80$ and $100 \%$ full contrast).

Other supplementary tests used in the ZVF study include: $1^{0}$ MPOD using the QuantifEye ${ }^{\circledR}$ heterochromatic flicker photometry (ZeaVision Chesterfield, MO), low- and high-contrast visual acuity, glare recovery, and photopic contrast sensitivity function using the Optec Plus (Stereo Optical, Chicago, IL, USA), auto fluorescence of the retina using the Optos 200Tx ${ }^{\circledR}$ (Optos, Dunfermline, Scotland, UK), color contrast sensitivity using the $6^{\circ}$ blue cone Chroma Test ${ }^{\circledR}$ Color, foveal shape discrimination (Retina Foundation of the Southwest, Dallas, TX, USA), and the National Eye Institute VFQ25 visual function questionnaire. Some of these results are mentioned parenthetically in the results. We evaluated the subset of 25 patients in the ZVF Study that received $8 \mathrm{mg}$ of dietary $\mathrm{Z}$ supplementation for 12 months. These 25 patients were retrospectively analyzed for central foveal dip in their macular pigment architecture, with five subjects demonstrating this dip phenomenon. In totality, 5/25 (20\%) of the subjects presented with a dip in their central macular pigment, which is consistent with previously published findings on central dip incidence $^{4-7}$ This article presents the MPOD ARIS scans and kinetic central macular visual field tests from four subjects because the fifth was excluded due to extremely poor image quality caused by dense bilateral nuclear cataracts. The cohort receiving $\mathrm{Z}$ supplementation was retrospectively investigated apart from the other two groups because of the scarcity of peer-reviewed publications focusing on $\mathrm{Z}$ supplementation.

\section{Results}

\section{Case I: Z32}

79-year old Caucasian male with bilateral AMD (OS worse than OD), extensive bilateral drusen, mild bilateral cataracts, and poor baseline contrast sensitivity function (Figure 4a) details impressive bilateral gradual resolution of his central scotoma; (Figure $4 b$ ) details restoration of central macular pigment peak; color vision also demonstrated improvement; finally, the subject noted modest gains in the subjective visual field questionnaire. 


\section{Baseline Image not available due to poor quality/technici an error}
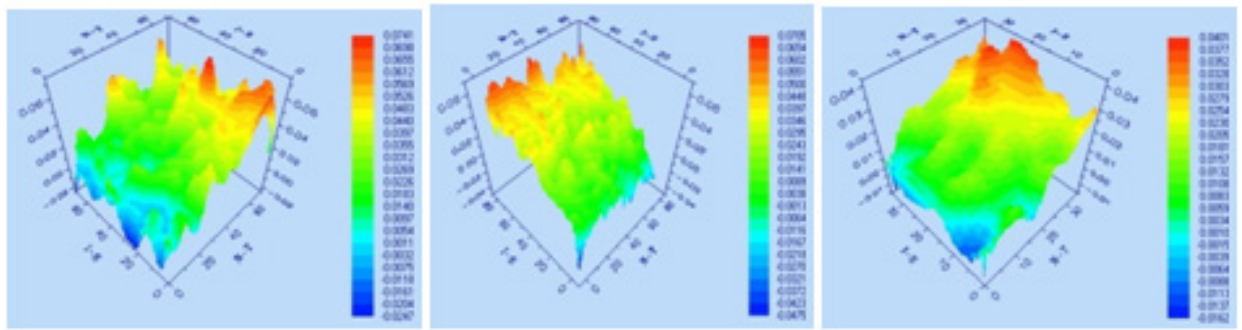

Figure 4a Case Z32 peak pigment at onset of study, 4 months, 8 months, and 12 months. The baseline measurement was unavailable due to poor quality.
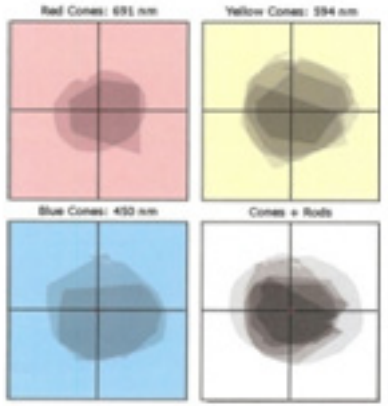
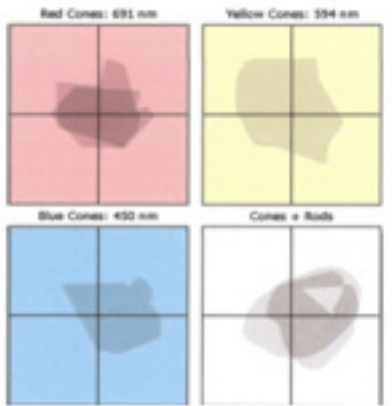
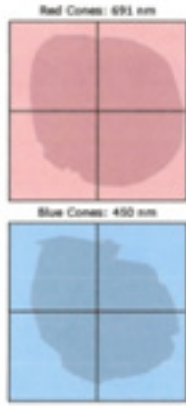
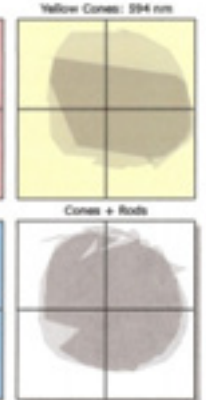
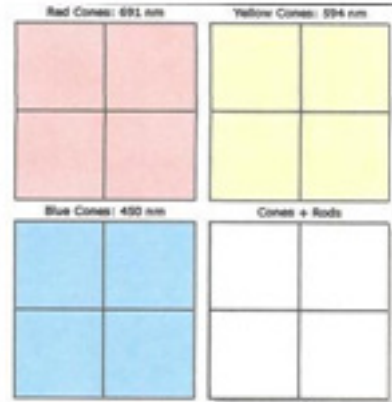

Figure 4b Case Z32 scotoma resolution at onset of study, 4 months, 8 months, and 12 months.

\section{Case 2: Z50}

50 -year old Caucasian female with progressively worsening night vision for approximately 30 years despite excellent visual acuity Figure 5 a shows clearing of her central foveal kinetic visual field defect while
Figure $5 \mathrm{~b}$ shows significant rebuilding of the central macular pigment peak. Z50 also showed reduced lipofuscin in autofluorescence images along with improved photopic contrast sensitivity function, Chroma Test color contrast sensitivity, and foveal shape discrimination.
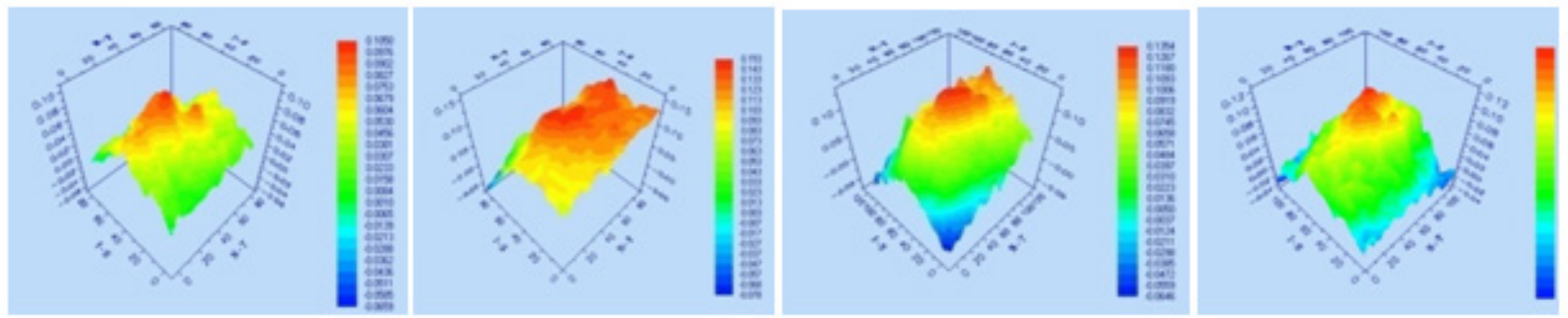

Figure 5 a Case Z50 peak pigment at onset of study, 4 months, 8 months, and 12 months.
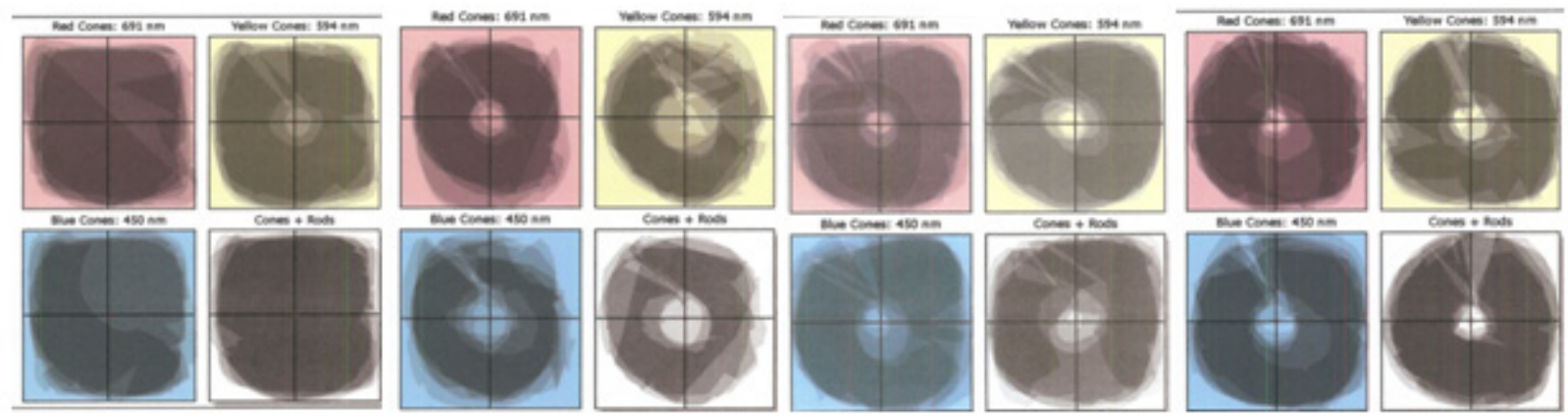

Figure 5b Case Z50 scotoma resolution at onset of study, 4 months, 8 months, and 12 months. 


\section{Case 3:Z55}

80-year old Caucasian male with OD AMD w/lipofuscin activity Figure 6a illustrates gradual but continuous improvement in his right kinetic visual field, which corresponds with similar gains in Amsler Grid tests and the visual field questionnaire. Figure $6 \mathrm{~b}$ shows development of better peak macular pigment architecture. Additionally, patient Z55's photopic contrast sensitivity function displayed dramatic improvement over the 12-month study.
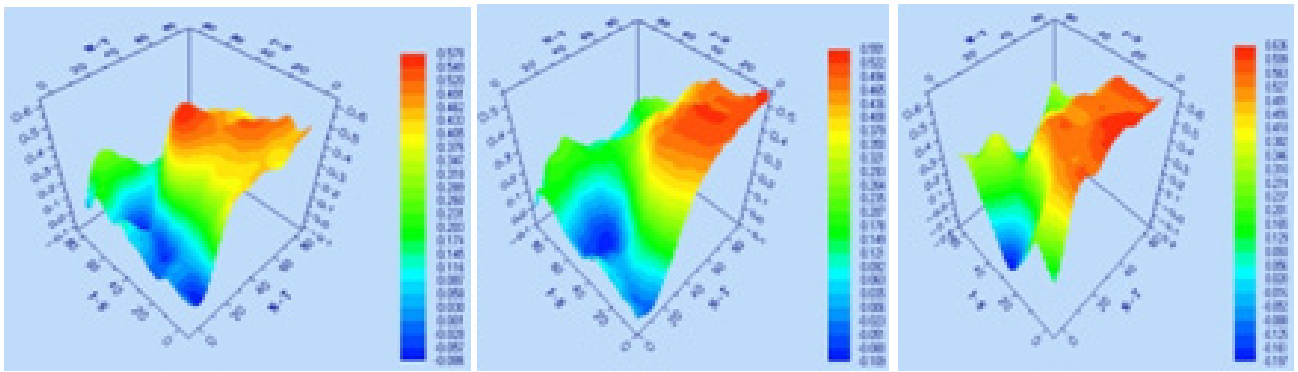

Final visit

Poor

Image

Quality

Figure 6a Case Z55 peak pigment at onset of study, 4 months, 8 months, and I 2 months.
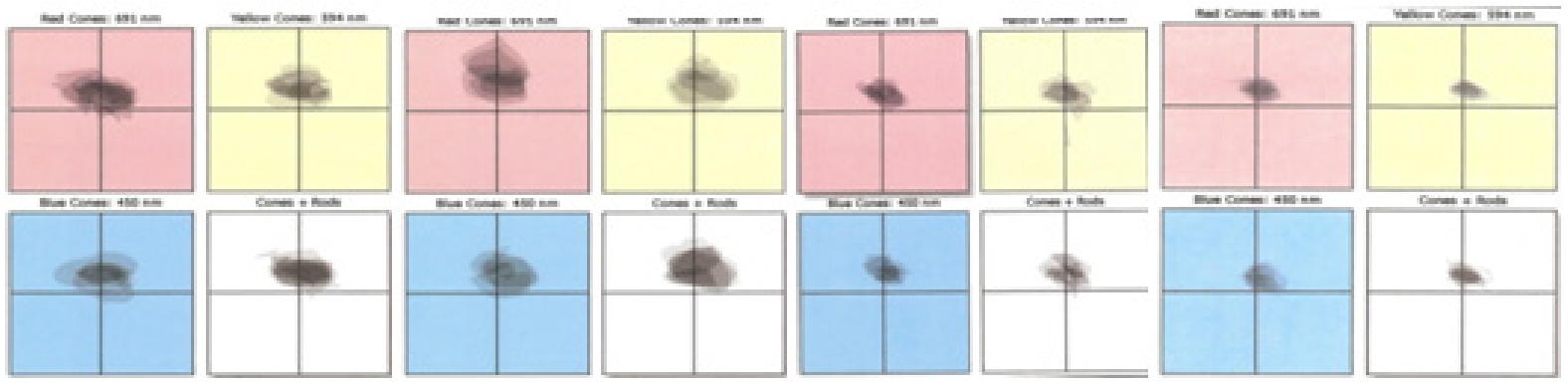

Figure 6b Case Z55 scotoma resolution at onset of study, 4 months, 8 months, and 12 months.

\section{Case 4: Z58}

87-year old Caucasian male w/ OD: combined AMD and early macula hole formation. Figure 7 a details significant reduction of his scotoma and Figure $7 \mathrm{~b}$ shows the development of a preferred central peak of macular pigment contrasting with the broader plateau-like distribution of the baseline image. Patient Z58 also exhibited increases in Amsler Grid and color vision test results. Notably, each Z-dip patient experienced improvement in one or more visual parameters: three of four subjects improved their color vision as measured by the $6^{0}$ blue cone color Chroma Test, two showed better contrast sensitivity function, two demonstrated faster glare recovery, and one experienced increased foveal shape discrimination.
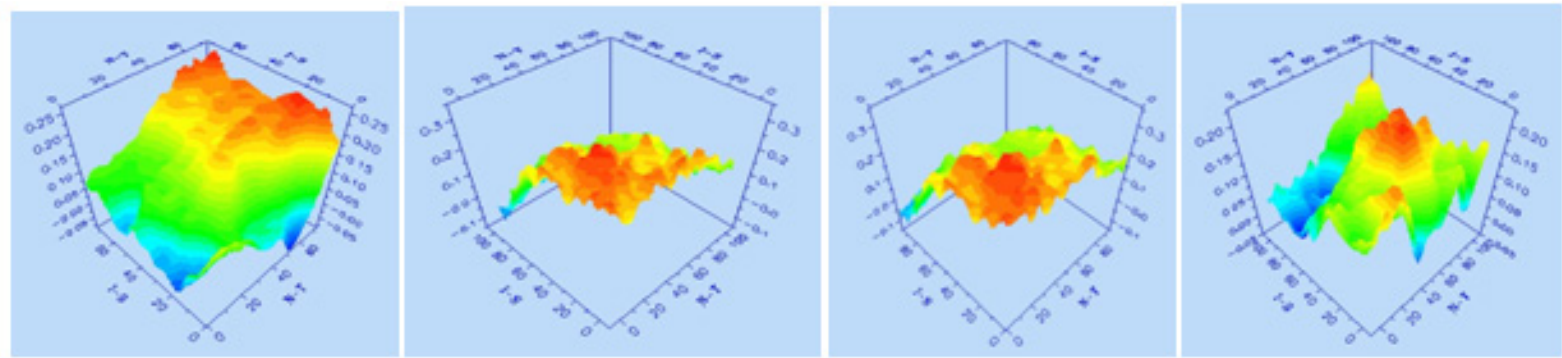

Figure 7a Case Z58 peak pigment at onset of study, 4 months, 8 months, and 12 months.

\section{Discussion}

There have been several attempts to identify $\mathrm{Mz}$ in human food sources but little consensus has been reached. ${ }^{18-23}$ Currently the balance of evidence suggests that $\mathrm{Mz}$ is present in the skin of a handful of marine species, though the quantities of Mz present in these sources is under dispute. Even the most optimistic data shows only $0.2 \mathrm{mg}$ of $\mathrm{Mz}$ present in an entire medium-sized trout weighing approximately
$200 \mathrm{~g} .{ }^{24}$ Considering a standard western diet, the trace amount of $\mathrm{Mz}$ in food sources makes consumption of dietary Mz functionally zero. Given Mz's key role in central macular health, the paucity of dietary Mz suggests an alternative source(s) of retinal Mz. This hypothesis is supported by undetectable Mz levels in serum unless $\mathrm{Mz}$ supplements have been recently consumed. Additionally, the macula is the only tissue in the human body containing stores of $\mathrm{Mz}^{25}$ This stands in contrast to $\mathrm{Z}$, which has been found throughout the body 
and has multiple functions, including photoprotective benefits within skin, ${ }^{26}$ aiding brain development in juveniles, ${ }^{27}$ maintaining cognitive function ${ }^{14}$ and slowing memory decline ${ }^{28}$ in adults.

It has been long suggested that the major source of macular $\mathrm{Mz}$ in primates is enzymatically-derived from L. ${ }^{10}$ The physiological mechanism of this process was not understood until recently when Bernstein et al., ${ }^{29}$ identified the enzyme that converts $\mathrm{L}$ to $\mathrm{Mz}$ as RPE65, or retinoid isomerohydrolase. RPE65 is located within the RPE layer and is vital for the conversion of all trans-retinyl esters to 11-cis-retinol, making it a key enzyme in the visual cycle occurring in photoreceptive cells. RPE65 has been well-researched but $\mathrm{Mz}$ isomerization is a secondary enzymatic activity that has not been studied extensively; the full text from Bernstein et al., ${ }^{29}$ has yet to be published. The identification of the central dip in macular pigment in patients with AMD is a relatively recent discovery; as such there are a very limited number of peer-reviewed articles investigating treatment options. The first study to investigate the effects of carotenoid supplementation on patients exhibiting the macular dip phenomenon was published by Nolan et al., ${ }^{30}$ in October 2016. Subjects were divided into three groups, each receiving a different ratio of carotenoid supplementation over an 8-week period. MPOD levels were measured at $0.25^{\circ}, 0.5^{\circ}, 1.0^{\circ}, 1.75^{\circ}$ and $3.0^{\circ}$ of retinal eccentricity by the method of heterochromatic flicker photometry. The cohort receiving $10 \mathrm{mg}$ $\mathrm{L}, 2 \mathrm{mg} \mathrm{Z}$, and $2 \mathrm{mg} \mathrm{Mz}$ produced statistically significant gains in MPOD at only the $0.25^{\circ}$ retinal eccentricity. Supplementation with 20 $\mathrm{mg} \mathrm{L}, 2 \mathrm{mg} \mathrm{Z}$, and zero Mz or 3mg L, $2 \mathrm{mg} \mathrm{Z}$, and $17 \mathrm{mg} \mathrm{Mz} \mathrm{did} \mathrm{not}$ produce statistically significant results at any eccentricity.
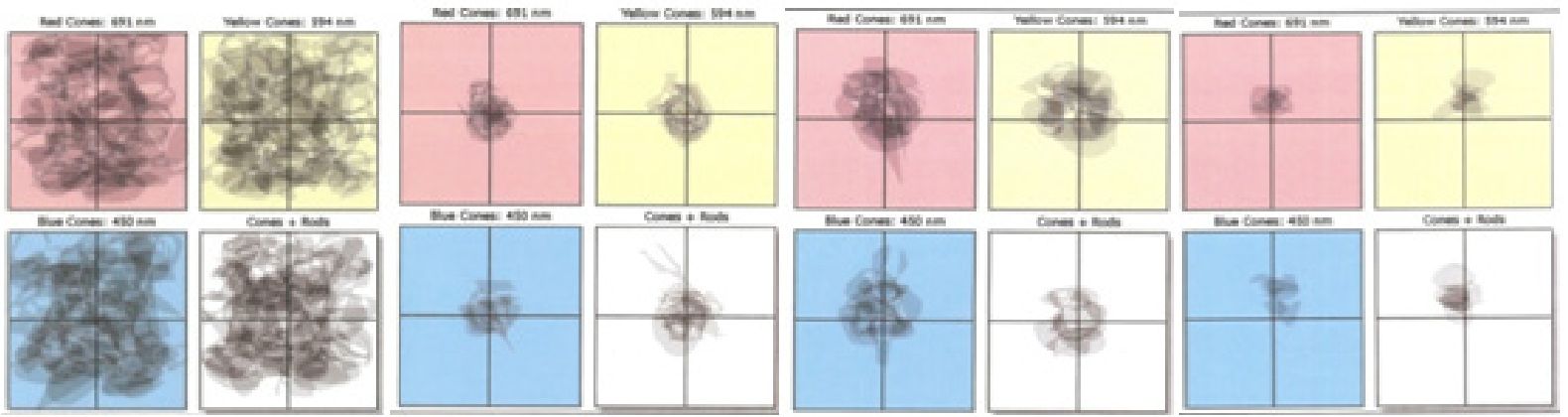

Figure 7b Case Z58 scotoma resolution at onset of study, 4 months, 8 months, and 12 months.

The most recent research on carotenoid supplementation comes from the 24-month longitudinal Central Retinal Enrichment Supplementation Trial (CREST). ${ }^{31}$ Subjects with early AMD were assigned one of two supplements: the AREDS2 formula or the AREDS2 formula plus $10 \mathrm{mg} \mathrm{Mz}$. The patients that received $\mathrm{Mz}$ supplementation showed no additional benefits in visual performance or macular pigment when compared to the subjects that received the traditional AREDS2 formula. Taken together, the respective papers by Nolan et al., ${ }^{30}$ and Akuffo et al., ${ }^{31}$ provide important insight in understanding how carotenoid supplementation affects AMD progression, but they ignore the effects of dietary $\mathrm{Z}$ in re-pigmenting the macula by using a small and non-variable supplemental dose of Z. Xanthophyll carotenoids were initially thought to be absorbed by passive diffusion, but identification of apical membrane transporters in intestinal enterocytes now point to an active absorption process. ${ }^{32}$ This is supported by research demonstrating competitive inhibition between carotenoids, ${ }^{33}$ though the details of this physiological mechanism are still being researched. Carotenoid bioavailability is a complex, multifactorial process influenced by the quantity and species of carotenoids consumed, whether carotenoids are consumed with fat and/or fiber, ${ }^{34}$ the presence of key vitamins and nutrients that act as co-factors ${ }^{35}$, serum HDL levels, body fat percentage, and endogenous carotenoid stores. ${ }^{36}$

Like Mz, $\mathrm{Z}$ is most highly concentrated in the central foveola, though very few studies have isolated dosing effects of dietary $\mathrm{Z}$ on retinal health. Research has demonstrated that carotenoid supplementation increases MPOD in patients with AMD, yet little is understood about re-pigmenting targeted regions within the macula. Furthermore, the handful of studies that have investigated carotenoid supplementation almost universally utilize a L:Z:Mz ratio of 10:2:0. This was the ratio used in the AREDS2 study ${ }^{35}$ and is based on the average ratio of dietary xanthophyll carotenoid consumption in the United States. Interestingly, the serum ratio of these carotenoids is $3: 1: 0,{ }^{37}$ yet no trial has ever supplemented at this ratio. Although the link between dietary carotenoid supplementation and reduced risk of ocular disease is robust, the interactions between the three macular carotenoids as well as their individual roles within specific areas of the retina (e.g. the foveola) remain unclear.

\section{Conclusion}

Supplementation with $\mathrm{Z}$ has been shown to improve visual performance and suppress retinal neovascularization that is characteristic of advanced $\mathrm{AMD}^{38}$ while yielding a lower cost-utility ratio than identical treatments without $\mathrm{Z}$ supplementation. ${ }^{39}$ The authors believe this paper provides the first evidence of resolution of a central dip in macular pigment density from dietary $Z$ supplementation. The ongoing debate over the efficacy of dietary $Z$ versus supraphysiologic/pharmacologic doses of $\mathrm{Mz}$ in re-pigmenting the macula remains open to further scientific inquiry, as the few investigations into $\mathrm{Mz}$ supplementation have produced contradictory results. ${ }^{30,31}$ These issues encompass the larger question of whether the two carotenoids ubiquitous in the human $\operatorname{diet}(\mathrm{L}$ and $\mathrm{Z}$ ) are sufficient to address age-related macular degeneration, or if $\mathrm{Mz}$ must be included as well. When considered in the context of recent publications such as the CREST study ${ }^{31}$ and Bernstein et al., ${ }^{29}$ the findings of this study justify further research into the role of $\mathrm{Z}$ versus $\mathrm{Mz}$ in re-pigmenting the foveola, particularly in patients that demonstrate a central dip in MPOD. These results warrant investigation into supplementation of $Z$ 
at higher dosages than the L:Z: Mz ratio of 10:2:0, which has become the de facto standard following the landmark AREDS2 study. ${ }^{35}$ The authors suggest a double-blind, randomized, placebo-controlled study that investigates higher doses of supplemental $\mathrm{Z}$, using the serum ratio of 3:1:0 as a minimum. Future prospective studies utilizing larger sample sizes and variable doses of $\mathrm{Z}$ would provide key insight into the relative roles of the three xanthophyll carotenoids in retinal health, regardless of underlying macular pigment architecture.

\section{Acknowledgements}

This article and the medical cases reported within represent further analysis of the published ZVF study (ZVF-FDA IND \#78, 973), which was approved by the Human Subjects and Research and Development IRB of the Department of Veterans Affairs. This work is supported by the Optometry/Ophthalmology sections of the Captain James A. Lovell Federal Health Care Center, a Department of Veterans Affairs and Department of Defense/US Navy Medical Center located in North Chicago, Illinois, USA

\section{Conflicts of interest}

SR is the global scientific research director of the Zeaxanthin Trade Association. DG is the Co-founder, Chairman and Chief Science Officer at ZeaVision. The remaining authors declare no conflicts of interest.

\section{Funding}

None.

\section{References}

1. Centers for Disease Control and Prevention, Common Eye Disorders, 2015.

2. Liew SH, Gilbert CE, Spector TD, et al. Central retinal thickness is positively correlated with macular pigment optical density. Exp Eye Res. 2006;82(5):915-920.

3. Trieschmann M, Spital G, Lommatzsch A, et al. Macular pigment quantitative analysis on auto fluorescence images. Graefes Archive for Clinical and Experimental Ophthalmology. 2003;241(12):1006-1012.

4. Kirby ML, Beatty S, Loane E, et al. A Central Dip in the Macular Pigment Spatial Profile Is Associated with Age and Smoking. Invest Ophthalmol Vis Sci. 2010;51(12):6722.

5. Dietzel M, Zeimer M, Heimes B, et al. The Ringlike Structure of Macular Pigment in Age-Related Maculopathy: Results from the Muenster Aging and Retina Study (MARS). Invest Ophthalmol Vis Sci. 2011;52(11):8016-8024.

6. Huntjens B, Ctori I. Variations and repeatability of macular pigment and its spatial profiles in south Asian and white subjects. Acta Ophthalmologica. 2014. p. 92.

7. Ctori I, Huntjens B. The Association between Foveal Morphology and Macular Pigment Spatial Distribution: An Ethnicity Study. Plos One. 2017;12(1):e0169520.

8. Neelam K, Ho H, Yip CC, et al. The Spatial Profile of Macular Pigment in Subjects From a Singapore Chinese Population. Invest Ophthalmol Vis Sci. 2014;55(4):2376-2383.

9. Sommerburg O, Keunen JE, Bird AC, et al. Fruits and vegetables that are sources for lutein and zeaxanthin: the macular pigment in human eyes. Br J Ophthalmol. 1998;82(8):907-910.
10. Bone RA, Landrum JT, Hime GW, et al. Stereochemistry of the human macular carotenoids. Invest Ophthalmol Vis Sci. 1993;34(6):20332040 .

11. Liu R, Wang T, Zhang B, et al. Lutein and Zeaxanthin Supplementation and Association With Visual Function in Age-Related Macular Degeneration. Invest Ophthalmol Vis Sci. 2015;56(1):252-258.

12. Bovier ER, Renzi LM, Hammond BR. A Double-Blind, PlaceboControlled Study on the Effects of Lutein and Zeaxanthin on Neural Processing Speed and Efficiency. PLoS One. 2014;9(9).

13. Johnson EJ. A possible role for lutein and zeaxanthin in cognitive function in the elderly. Am J Clin Nutr. 2012;96(5):1161S-5S.

14. Stringham NT, Holmes PV, Stringham JM. Supplementation with macular carotenoids reduces psychological stress, serum cortisol, and sub-optimal symptoms of physical and emotional health in young adults. Nutr Neurosci. 2017;15:1-11.

15. Ying C, Chen L, Wang S, et al. Zeaxanthin ameliorates high glucoseinduced mesangial cell apoptosis through inhibiting oxidative stress via activating AKT signalling-pathway. Biomed Pharmacother. 2017;90:796-805.

16. Ma L, Liu R, Du JH, et al. Lutein, Zeaxanthin and Meso-zeaxanthin Supplementation Associated with Macular Pigment Optical Density. Nutrients. 2016;8(7):426.

17. Richer SP, Stiles W, Graham-Hoffman K, et al. Randomized, doubleblind, placebo-controlled study of zeaxanthin and visual function in patients with atrophic age-related macular degeneration: the Zeaxanthin and Visual Function Study (ZVF) FDA IND \#78, 973. Optometry. 2011;82(11):667-680.e6.

18. Maoka T, Arai A, Shimizu M, et al. The first isolation of enantiomeric and Meso-zeaxanthin in nature. Comp Biochem Physiol B. 1986;83(1):121-124.

19. Johnson EJ, Neuringer M, Russell RM, et al. Nutritional Manipulation of Primate Retinas, III: Effects of Lutein or Zeaxanthin Supplementation on Adipose Tissue and Retina of Xanthophyll-Free Monkeys. Invest Ophthalmol Vis Sci. 2005;46(2):692-702.

20. Nolan JM, Meagher K, Kashani S, et al. What is meso-zeaxanthin, and where does it come from? Eye (Lond). 2013;27(8):899-905.

21. Bernstein PS, Johnson EJ, Neuringer M, et al. Comment on: What is meso-zeaxanthin, and where does it come from? Eye. 2013;28(2):240242 .

22. Nolan JM, Meagher K, Kashani S, et al. Response to Bernstein et al. Eye. 2013;28(2):242-244.

23. Nolan JM, Beatty S, Meagher KA, et al. Verification of MesoZeaxanthin in Fish. J Food Process Technol. 2014;5(6):335.

24. Prado-Cabrero A, Beatty S, Stack J, et al. Quantification of zeaxanthin stereoisomers and lutein in trout flesh using chiral high-performance liquid chromatography-diode array detection. J Food Compost Anal. 2016;50:19-22.

25. Khachik F, de Moura FF, Zhao DY, et al. Transformations of Selected Carotenoids in Plasma, Liver, and Ocular Tissues of Humans and in Nonprimate Animal Models. Invest Ophthalmol Vis Sci. 2002;43(11):3383-3392.

26. Roberts RL. Lutein, Zeaxanthin, et al. American Journal of Lifestyle Medicine. 2013;7(3):182-185.

27. Henriksen BS, Chan GM. Importance of Carotenoids in Optimizing Eye and Brain Development. J Pediatr Gastroenterol Nutr. 2014;59(5): 552. 
28. Hoffmann K, Richer S, Wrobel J, et al. A Prospective Study of NeuroCognitive Enhancement with Carotenoids in Elderly Adult Males with Early Age Related Macular Degeneration. Ophthalmology Research: An International Journal. 2015;4(1):1-8.

29. Bernstein PS, Shyam R, Gorusupudi A, Nelson K, Horvath M (2017) RPE65 Has a Secondary Activity as the Lutein to meso-Zeaxanthin Isomerase in the Vertebrate Eye. In: Proceedings from 2017 ARVO Annual Meeting; Baltimore, USA: Maryland; 2017.

30. Nolan JM, Akkali MC, Loughman J, et al. Macular carotenoid supplementation in subjects with atypical spatial profiles of macular pigment. Exp Eye Res. 2012;101:9-15.

31. Akuffo KO. Macular carotenoid supplementation and visual function in early age-related macular degeneration, 2016.

32. During A, Hussain MM, Morel DW, et al. Carotenoid uptake and secretion by CaCo-2 cells. J Lipid Res. 2002;43(7):1086-1095.

33. Wang Y, Illingworth DR, Connor SL, et al. Competitive inhibition of carotenoid transport and tissue concentrations by high dose supplements of lutein, zeaxanthin and beta-carotene. Eur J Nutr. 2010;49(6):327336 .

34. Priyadarshani AMB. A review on factors influencing bioaccessibility and bioefficacy of carotenoids. Critical Reviews in Food Science and Nutrition. 2015;57(8):1710-1717.
35. Chew EY, Clemons TE, Agrón E, et al. Effect of Omega-3 Fatty Acids, Lutein/Zeaxanthin, or Other Nutrient Supplementation on Cognitive Function. JAMA. 2015;314(8):791-801.

36. Kiokias S, Proestos C, Varzakas T. A Review of the Structure, Biosynthesis, Absorption of Carotenoids-Analysis and Properties of their Common Natural Extracts. Current Research in Nutrition and Food Science Journal. 2015;4(1):25-37.

37. Abdel-Aal el-SM, Akhtar H, Zaheer K, Ali R, et al. Dietary Sources of Lutein and Zeaxanthin Carotenoids and Their Role in Eye Health. Nutrients. 2013;5(4):1169-1185.

38. Rosen R, Vagaggini T, Chen Y, et al. Zeaxanthin Inhibits HypoxiaInduced VEGF Secretion by RPE Cells through Decreased Protein Levels of Hypoxia-Inducible Factors-1 $\alpha$. Biomed Res Int. 2015. p. $687-386$.

39. Olk RJ, Peralta E, Gierhart DL, et al. Triple combination therapy and zeaxanthin for the treatment of neovascular age-related macular degeneration: an interventional comparative study and costeffectiveness analysis. International Journal of Retina and Vitreous. 2015;1:22.

40. Schalch W. Possible contribution of lutein and zeaxanthin, carotenoids of the macula lutea, to reducing the risk for age-related macular degeneration: a review. HKJ Ophthalmology. 2000;4(1). 